\title{
Neuromechanical activation of triceps surae muscle remains altered at 3.5 years following open surgical repair of acute Achilles tendon rupture
}

\author{
Markus Wenning ${ }^{1,2}$ - Marlene Mauch ${ }^{1} \cdot$ Albrecht Heitner $^{1} \cdot$ Johannes Lienhard ${ }^{3} \cdot$ Ramona Ritzmann $^{1} \cdot$ Jochen Paul $^{1}$
}

Received: 15 October 2020 / Accepted: 17 February 2021 / Published online: 3 March 2021

(c) The Author(s) 2021

\begin{abstract}
Purpose To assess whether the neuromuscular activation pattern following Achilles tendon rupture and repair may contributes to the observable functional deficits in this severe and increasingly frequent injury.

Methods In this study, the neuromuscular activation using surface EMG of $n=52$ patients was assessed during a battery of functional performance tasks to assess potential alterations of muscular activation and recruitment. We analyzed the injured leg vs. the contralateral healthy leg at a mean of 3.5 years following open surgical repair. The testing battery included isokinetic strength testing, bipedal and single-legged heel-rise testing as well as gait analysis.

Results During isokinetic testing, we observed a higher activation integral for all triceps surae muscles of the injured side during active dorsiflexion, e.g., eccentric loading on the injured leg, while concentric plantarflexion showed no significant difference. Dynamic heel-rise testing showed a higher activation in concentric and eccentric loading for all posterior muscles on the injured side (not significant); while static heel-rise for $10 \mathrm{sec}$. revealed a significantly higher activation. Further analysis of frequency of fast Fourier-transformed EMG revealed a significantly higher median frequency in the injured leg. Gait analysis revealed a higher pre-activation of the tibialis anterior before ground contact, while medial and lateral gastrocnemius muscles of the injured leg showed a significantly higher activation during push-off phase.

Conclusions The results of this study provide evidence on the neuromuscular changes 3.5 years following open surgical Achilles tendon repair. These complex neuromuscular changes are manifested to produce the maximum force output whilst protecting the previously injured tendon. The observed alterations may be related to an increased recruitment of type II muscle fibers which could make the muscles prone to fatigue.
\end{abstract}

Level of evidence III.

Keywords Achilles tendon rupture $\cdot \mathrm{EMG} \cdot$ Neuromuscular activation $\cdot$ Functional performance testing

Abbreviations
$\begin{array}{ll}\text { TA } & \text { Tibialis anterior muscle } \\ \text { GM } & \text { Gastrocnemius medialis muscle } \\ \text { GL } & \text { Gastrocnemius lateralis muscle }\end{array}$

Markus Wenning

research@ rennbahnklinik.ch

Rennbahnklinik, Muttenz, Basel, Switzerland

2 Department of Orthopedic and Trauma Surgery, Faculty of Medicine, University Medical Center Freiburg, Albert-Ludwigs University of Freiburg, Freiburg, Germany

3 Department of Sport and Sport Science, Biomechanics and Motor Control, Albert-Ludwigs University of Freiburg, Freiburg, Germany
SOL Soleus muscle

EMG Electromyogram/-graphy

\section{Introduction}

Achilles tendon rupture is a severe injury to any athlete and, regardless of the treatment, only about $70 \%$ achieve a return to preinjury athletic level [28]. Furthermore, of those that return, many show a reduced performance level in the first year after returning to sport or even longer [28, 29].

The reasons behind these performance deficits may primarily be found in the tendon healing itself; however, the neuromuscular impact on these deficits has rarely been investigated. Primarily an increased muscle activity and activation ratio on the injured side has been described 
during various tasks $[17,20,26]$. Further analysis has shown that the repaired Achilles tendon is compliant, but that greater strain results in less effective energy storage, e.g., during hopping tasks [20]. Additionally, studies reported less plantarflexion and increased dorsiflexion during gait which was linked to tendon stiffness and elongation $[1,9,21]$. An altered neuromuscular activation pattern reflected by the augmented tibialis anterior muscle and soleus muscle co-contraction index has been shown to be present [9]. Thus, it may be assumed that these alterations of neuromuscular activation contribute to the persisting mid- and long-term functional performance deficits [17]. While the structural changes observed in the tendon's properties have been well investigated [22, 23], current literature underscores the importance of taking additional neuromuscular alterations into account when investigating persisting performance deficits after Achilles tendon rupture $[14,17,25]$.

Thus, the aim of this investigation was to provide evidence for mid-term neuromechanical alterations following open Achilles tendon repair using a combination of performance tests in a large and representative cohort. Deducted from the literature [17, 20], it was hypothesized that muscular activation of plantar flexors would be higher in the injured leg compared to the healthy leg across all tasks, while neuromuscular activation pattern would be adapted to decrease excessive load on the ruptured tendon.

\section{Materials and methods}

This is the second part of results from a large and multivariate cross-sectional comparative study. The study was performed in accordance with the Declaration of Helsinki, it was approved by the local ethics committee (EKNZ 201702206) and all participants declared informed consent prior to inclusion.

Mid-term neuromechanical activation deficits were assessed during functional performance testing of $n=52$ patients which had previously undergone open surgical repair for acute Achilles tendon rupture. Inclusion criteria were acute Achilles tendon open repair in one of the two centers involved in the study, fewer than ten days after rupture, male gender and age $<60$ years. Exclusion criteria were other injuries to the lower extremities, injury to the contralateral tendon, re-rupture, neurological impairments and diabetes mellitus. All tests were performed in a single session and supervised by two examiners experienced in biomechanical testing. The functional performance testing including strength testing, heel-rise test and gait analysis has been discussed separately [32].

\section{Patients}

A total of $n=138$ patients from two centers meeting the inclusion/exclusion criteria were assessed for eligibility. In summary, we were able to include $n=52$ male patients at a mean follow-up of $3.45 \pm 1.4$ years, with a mean height of $1.81 \pm 0.6 \mathrm{~m}$ and mean weight of $88.7 \pm 11.7 \mathrm{~kg}$. Mean age at surgery was $41 \pm 9.5$ years with the dominant leg (the leg one would jump with) injured in $52 \%$ of cases. The details of the recruitment process have been described before.

All patients had received physical therapy and followed a standardized rehabilitation protocol. All patients had finished their rehabilitation before enrollment in the study and had returned to any type sportive activities including running.

\section{Testing protocol}

The details of the testing protocol have been described before [32]. The testing protocol included three different components:

(A) an isokinetic strength testing of plantarflexion and dorsiflexion (Humac Norm, CSMi, Stoughton, MA, USA) according to the literature [2]. The testing was performed with the patient placed in prone position in full knee extension. Three warm-up trials were followed by two sets of five repetitions at maximum effort. The protocol of concentric-concentric contractions at $30 \%$ angular speed in the full range of motion (ROM) was chosen due to its high test-retest reliability [2] and validity [18].

(B) Heel-rise testing using a novel approach in a markerbased 3D motion analysis laboratory (Vicon Motion System Ltd., Oxford, UK) and four different testing modalities: single-legged and bipedal testing, each during two different tasks: static testing with the patient staying at maximum heel-rise height for $10 \mathrm{~s}$ and dynamic testing with five repetitive heel-rises at moderate speed.

(C) Gait analysis using the same marker-based system for triggering EMG analysis via an integrated force plate (Kistler AG, Winterthur, Switzerland). Surface EMG $\mathrm{Ag} / \mathrm{AgCl}$ electrodes (Blue Sensor Typ N, Ambu GmbH, Bad Nauheim, Deutschland) were placed on both shanks according to the SENIAM guidelines [12]. Transmission was realized using wireless EMG signaling (myon AG, Schwarzenberg, Switzerland) at a sampling rate of $2 \mathrm{kHz}$. EMG signal was collected bilaterally from the tibialis anterior muscle (TA), Gastrocnemius lateralis (GL) and medialis (GM) and soleus (SOL) muscle. 
Details of the performance testing during EMG analysis were as follows:

Isokinetic testing Of the ten repetitions in total, the first one of each set was excluded to achieve a comparable preconditioning, the mean of the best four of the remaining eight repetitions was included in the analysis.

Heel-rise During heel-rise testing the time normalization was performed using the marker-based motion signal and the maximum and minimum value of the heel-marker defining the phases during dynamic heel-rise testing. For the analysis of muscle activity, we defined the first $2 \mathrm{~s}$ as the starting phase, a stable isometric phase of $6 \mathrm{~s}$ before entering into the final phase from seconds 8-10. We implemented a Fast Fourier analysis to measure muscle fatigue during the static condition.

Gait analysis During gait analysis, the pre-activation was defined as the integral activity $100 \mathrm{~ms}$ before ground contact. The Braking phase was defined from ground contact to when the anteroposterior force component at the force plate was crossing zero. Push-off phase was defined from the same point to toe-off. Mean value of six repetitions was calculated. Co-activation was defined as the normalized EMG of the plantarflexors (SOL, GM, GL) divided by the normalized EMG of the dorsiflexor (TA) [24].

\section{EMG signaling and processing}

Bipolar $\mathrm{Ag} / \mathrm{AgCl}$ surface electrodes (Ambu Blue Sensor P, Ballerup, Denmark, diameter $9 \mathrm{~mm}$, center-to-center distance $34 \mathrm{~mm}$ ) were placed over the tibialis anterior muscle (TA), gastrocnemius medialis muscle (GM), gastrocnemius lateralis muscle (GL) and soleus muscle (SOL) muscles of both legs. Procedures were executed according to SENIAM [12]. Post processing was performed using Nexus 2.7 (Oxford Metrics, Ltd., Oxford, UK), proEMG 2.0 (Prophysics, Kloten, Switzerland) and MathLabR2017b (The Mathworks Inc., Natick, MA, USA). Before statistical analysis, we applied a fourth-order butterworth bandpass filter $(20-500 \mathrm{~Hz})$ to a rectified EMG signal. EMG signal was integrated, timenormalized and normalized to MVC conditions according to the literature $[5,33]$ using the peak signal during maximum isokinetic contraction for amplitude normalization. To assess the firing frequency, a fast Fourier transformation (FFT) for spectral analysis was performed according to the literature with rectangular windowing and $2^{n}$ sampling of the median frequency $[6,30]$.

\section{Statistical analysis}

The statistical analysis was performed using SPSS 24 (SPSS inc., Chicago, USA). Graphical display was realized using Veusz v. 3.0.1 (Veusz Group by Sanders et al., GNU-licensed, 2018).
An a priori sensitivity analysis assuming a power of 0.8 and an alpha error of 0.05 and a population of $n=52$ resulted in a required effect size of 0.39 . All variables were normally distributed (Shapiro-Wilk). The absolute values of the subgroups and the corresponding statistics are available in the online supplement. We performed a single-factor ANOVA with the factor side. For analyzing static heel-rise, we used a two-factor repeated-measures ANOVA with the three-level factor time and the two-level factor side with a Greenhouse-Geisser correction because Mauchly's test of sphericity was significant for the factor time. Additionally, a post hoc analysis of pairwise comparison for the three-level factor time was performed. Generally, due to the explorative approach, the level of significance was kept at $p<0.05$ for all tests, except post hoc analysis of rm-ANOVA where a Bonferroni-Holm correction of the alpha level was applied.

\section{Results}

\section{Isokinetic testing}

Isokinetic testing showed significant differences during eccentric activation when performing dorsiflexion with all muscles of the triceps surae complex showing a significantly higher activation rate on the operated side (Table 1, Fig. 1). The strongest effect was visible for gastrocnemius medialis muscle activation with an activation rate which was a relative $35 \%$ higher compared to the healthy leg $(F(1,94)=12,450$, $p<0.001)$. The tibialis anterior muscle activation was comparable in both conditions. During dorsiflexion the activation of soleus muscle was nearly twice the amount, when compared

Table 1 EMG activation during single-legged isokinetic testing in concentric-concentric mode

\begin{tabular}{llrrl}
\hline Parameter & Muscle & Operated (\%MVC) & \multicolumn{1}{l}{$\begin{array}{l}\text { Non- } \\
\text { operated } \\
(\% \mathrm{MVC})\end{array}$} & ANOVA \\
\hline Plantarflexion & TA & $5.3 \pm 2.1$ & $5.5 \pm 2.0$ & n.s. \\
& GL & $41.1 \pm 6.5$ & $40.5 \pm 5.5$ & n.s. \\
& GM & $39.8 \pm 6.2$ & $41.1 \pm 5.7$ & n.s. \\
& SOL & $37.3 \pm 5.4$ & $35.4 \pm 7.2$ & n.s. \\
Dorsiflexion & TA & $41.7 \pm 4.2$ & $41.7 \pm 6.2$ & n.s. \\
& GL & $\mathbf{7 . 2} \pm \mathbf{2 . 3}$ & $\mathbf{6 . 0} \pm \mathbf{2 . 1}$ & $\boldsymbol{p}=\mathbf{0 . 0 1 2}$ \\
& GM & $\mathbf{8 . 1} \pm \mathbf{3 . 3}$ & $\mathbf{6 . 0} \pm \mathbf{2 . 4}$ & $\boldsymbol{p}<\boldsymbol{0 . 0 0 1}$ \\
& SOL & $\mathbf{1 8 . 5} \pm \mathbf{8 . 7}$ & $\mathbf{1 5 . 3} \pm \mathbf{5 . 1}$ & $\boldsymbol{p}=\mathbf{0 . 0 3}$ \\
\hline
\end{tabular}

Bold values represent significant difference

$T A$ tibialis anterior muscle; $G L$ gastrocnemius lateralis muscle; $G M$ M. gastrocnemius medialis muscle; SOL soleus muscle; $\% M V C$ in $\%$ of the peak value during maximum voluntary isokinetic contraction; ANOVA single-factor ANOVA, factor: side, mean \pm standard deviation 
Fig. 1 Muscle activation during isokinetic strength testing showing the increased muscular activation of the posterior muscle group during dorsiflexion. $* p<0.05$

\section{EMG-Activity during isokinetic strength testing}

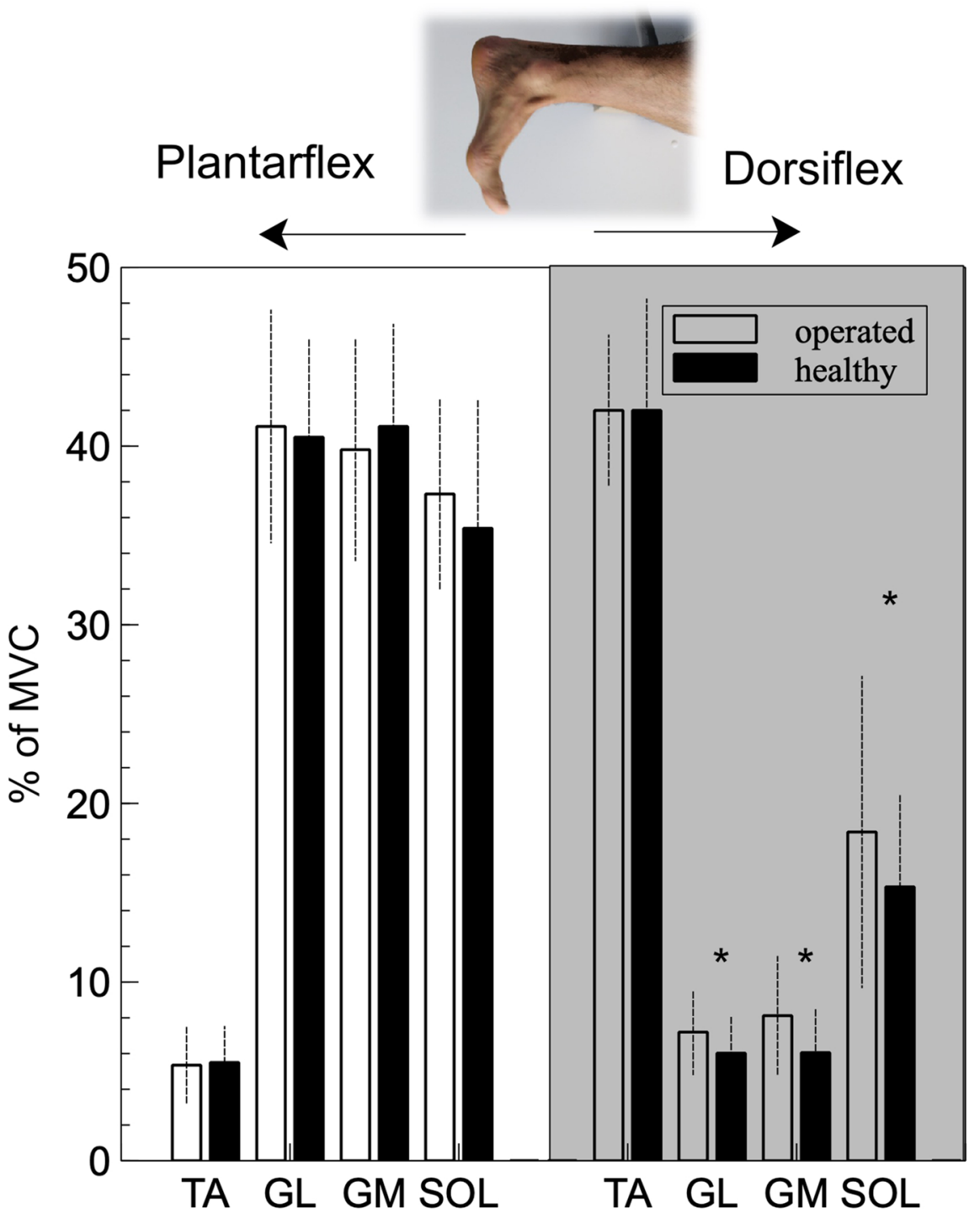

to the two gastrocnemius muscles; however, this difference was not significant.

\section{Heel-rise testing: dynamic}

The results of the dynamic single-leg heel-rise testing are displayed in Table 2 and Fig. 2; additionally in Table 2, the results of bipedal heel-rise testing are displayed. The activation rate of all three posterior muscles was higher when compared to the healthy leg; however, only lateral gastrocnemius muscle during eccentric contraction ("going down") showed a significant difference $(p<0.05$, $F(1,84)=5408)$. Activation of tibialis anterior muscle was comparable during concentric and eccentric contraction, while activation of triceps surae muscles was two-three times higher during concentric contraction, which resulted in a higher co-contraction index compared to concentric contraction.

\section{Heel-rise testing: static}

Maximum amplitude and height during static heel-rise were significantly reduced in the injured leg. When performing single-leg static heel-rise testing, there were significant differences for time and side as shown in Fig. $3 a+b$ and Table 3. The injured side showed a significantly higher integral EMG activity across all time intervals compared to the healthy side and the rm-ANOVA also revealed a significant effect of the factor time. The spectral analysis revealed a significantly higher median frequency in the 
Table 2 EMG activation during dynamic heel-rise

\begin{tabular}{llccc}
\hline Parameter & Muscle & Operated (\%MVC) & $\begin{array}{c}\text { Non-operated } \\
(\% \text { MVC })\end{array}$ & ANOVA \\
\hline Single-leg dynamic & TA conc & $3.1 \pm 1.5$ & $3.3 \pm 2.5$ & n.s. \\
& TA ecc & $2.3 \pm 1.1$ & $2.4 \pm 2.1$ & n.s. \\
& GL conc & $31.4 \pm 9.5$ & $27.8 \pm 12.9$ & n.s. \\
& GL ecc & $12.1 \pm 4.8$ & $10.1 \pm 6.4$ & n.s. \\
& GM conc & $33.8 \pm 9.5$ & $32.7 \pm 19.9$ & n.s. \\
& GM ecc & $15.7 \pm 6.3$ & $14.2 \pm 7.5$ & n.s. \\
& SOL conc & $33.1 \pm 11.4$ & $31.9 \pm 18.5$ & n.s. \\
& SOL ecc & $14.5 \pm 6.2$ & $13.4 \pm 8.1$ & n.s. \\
Bipedal dynamic & TA conc & $2.7 \pm 1.4$ & $2.8 \pm 2.0$ & n.s. \\
& TA ecc & $4.1 \pm 3.2$ & $4.2 \pm 4.1$ & n.s. \\
& GL conc & $25.1 \pm 9.4$ & $21.6 \pm 11.8$ & n.s. \\
& GL ecc & $\mathbf{6 . 2} \pm \mathbf{2 . 9}$ & $\mathbf{4 . 7} \pm \mathbf{3 . 0}$ & $\boldsymbol{p}=\mathbf{0 . 0 2}$ \\
& GM conc & $30.8 \pm 14.5$ & $28.3 \pm 18.3$ & n.s. \\
& GM ecc & $9.9 \pm 4.5$ & $8.7 \pm 4.4$ & n.s. \\
& SOL conc & $25.6 \pm 10.4$ & $21.7 \pm 12.4$ & n.s. \\
& SOL ecc & $7.4 \pm 4.1$ & $5.8 \pm 3.9$ & n.s. \\
\hline
\end{tabular}

Bold values represent significant difference

$T A$ tibialis anterior; $G L$ gastrocnemius lateralis muscle; $G M$ gastrocnemius medialis muscle; $S O L$ soleus muscle; conc. concentric phase; ecc. eccentric phase; \%MVC in \% of the peak value during maximum voluntary isokinetic contraction; ANOVA single-factor ANOVA, factor: side, mean \pm standard deviation; n.s. non-significant

injured leg compared to the healthy side during all time intervals for medial and lateral gastrocnemius muscle with strong effect sizes.

\section{Gait analysis: EMG}

Gait analysis showed a significantly lower pre-activation $100 \mathrm{~ms}$ before ground contact for tibialis anterior muscle of the injured leg as displayed in Fig. 4. The co-contraction index of the injured ankle's dorsi- vs. plantarflexors was, therefore, 1.05 compared to the 1.35 of the healthy contralateral leg. During braking and push-off phase, the lateral gastrocnemius muscle showed a significantly higher muscle activation on the injured side and, during push-off phase, this was also the case for the medial gastrocnemius muscle (Table 4).

\section{Discussion}

The most important finding of this study was that during eccentric loading and isometric contraction, there is a higher activation of the triceps surae muscle complex on the injured side compared to the healthy contralateral side. Furthermore, the frequency analysis showed a higher recruitment on the injured side, which could be related to the increased activation of fast-twitch fibers. Finally, the activation pattern during gait revealed a significantly lower pre-activation of the tibialis anterior muscle before ground contact; while during the braking phase, there was a significantly higher activation of the lateral gastrocnemius muscle. During push-off phase, the activation of medial and lateral gastrocnemius muscles was also significantly higher, while soleus muscle activation was not altered.

\section{Isokinetic testing}

While the recruitment pattern during active concentric plantarflexion was not altered between the legs, there was a significant difference during active dorsiflexion for all three posterior muscles (Fig. 1). This increased muscular activation, however, does not lead to a reduced maximum dorsiflexion torque, but may in part be the reason why maximum peak torque angle during dorsiflexion is altered [32]. Moreover, the increased activation of all triceps surae muscles suggests that "active facilitation" during dorsiflexion is not observable following Achilles tendon rupture [13, 20].

The causal interpretation of this observation requires further analysis since, among others, we suggest that the following factors may contribute: (1) maximum concentric voluntary activation may exceed the side-to-side differences and, therefore, mask potential differences related to muscular activation that otherwise would support the continuously increased general muscular activation [26, 33]; (2) the increased activation during dorsiflexion may be caused by an altered spinal reflex path due to impaired afference from the repaired tendon itself [1]; or (3) there is persisting co-contraction as a preventive measure to limit excessive e.g. ballistic 
Fig. 2 Muscle activation during single-legged dynamic heel-rise showing an increased integral muscular activity on the operated leg

\section{Single-Leg Dynamic Heel-Rise}

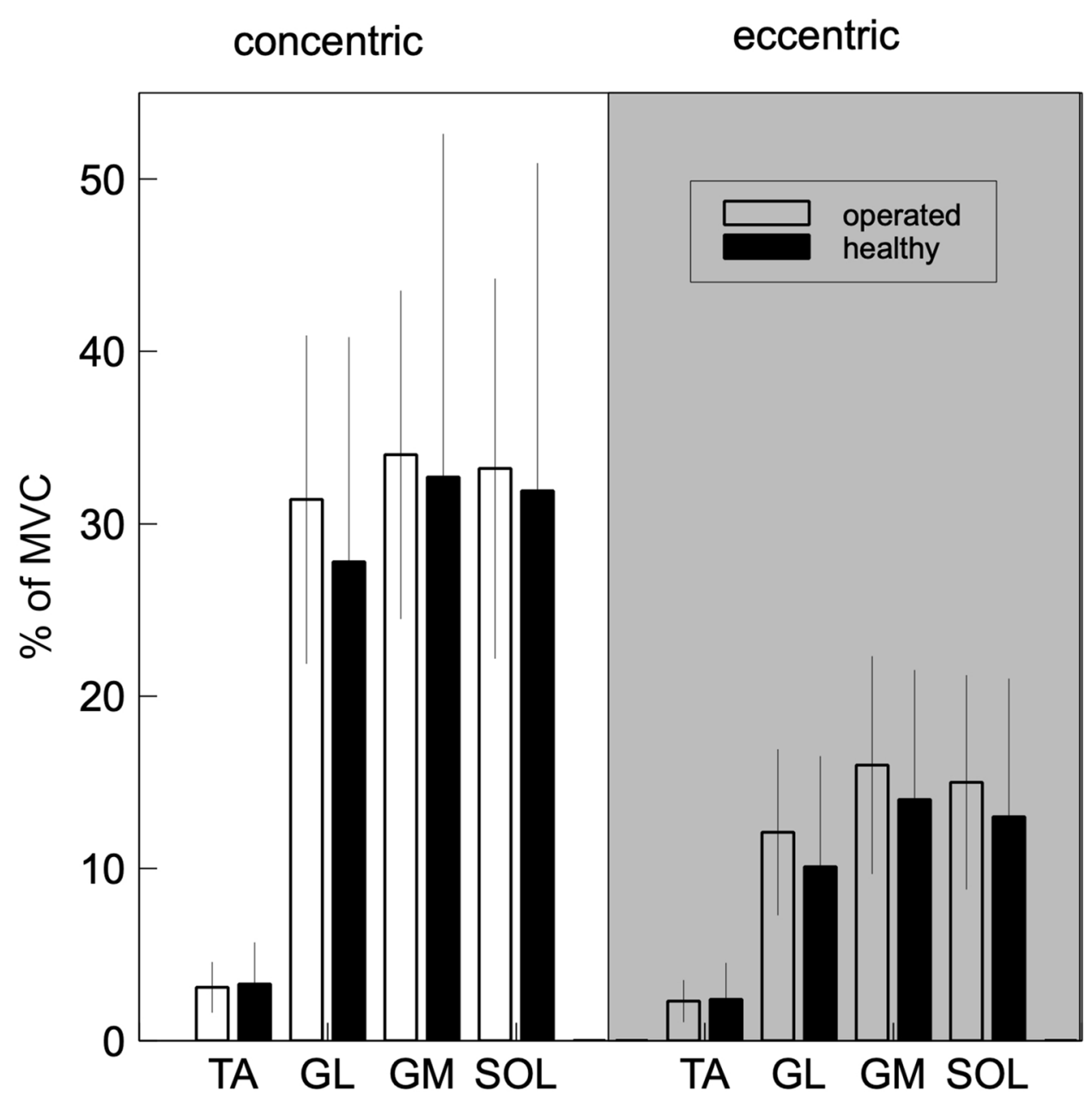

contraction and subsequent tendon elongation. As discussed by other authors before, this co-contraction may prevent the tendon from explosive, eccentric loading, even though this activation pattern increases net tendon strain [4, 7, 27, 33].

$\mathrm{McHugh}$ et al. suggested that the increase in activation may be attributed to the decrease in muscle fiber length and in their study, this increase in activation was pronounced when there was a significant weakness during plantarflexion [17].

\section{Dynamic heel-rise testing}

Furthermore, during eccentric contraction in dynamic heelrise testing (e.g., "going downward"), there is an increased muscular activation as it has been described before even in healthy subjects comparable to our results [11]. These findings are in line with McHugh et al. where an increased muscular activation was necessary to perform the same motor task [17, 34]. Using a different setup, Zellers et al. found a comparable, yet statistically significant, increase in plantarflexor activation $100 \mathrm{~ms}$ before landing from a jump, which was attributed to controlling dorsiflexion and stiffing the joint prior to eccentric load [34]. The same pattern was observed during bipedal testing.

Interestingly, even though the weight in this task is partially shifted away from the injured side and consequently less force is required, the muscular activation level is still increased. 


\section{EMG frequency (FFT)}

a during single-leg static Heel-Rise

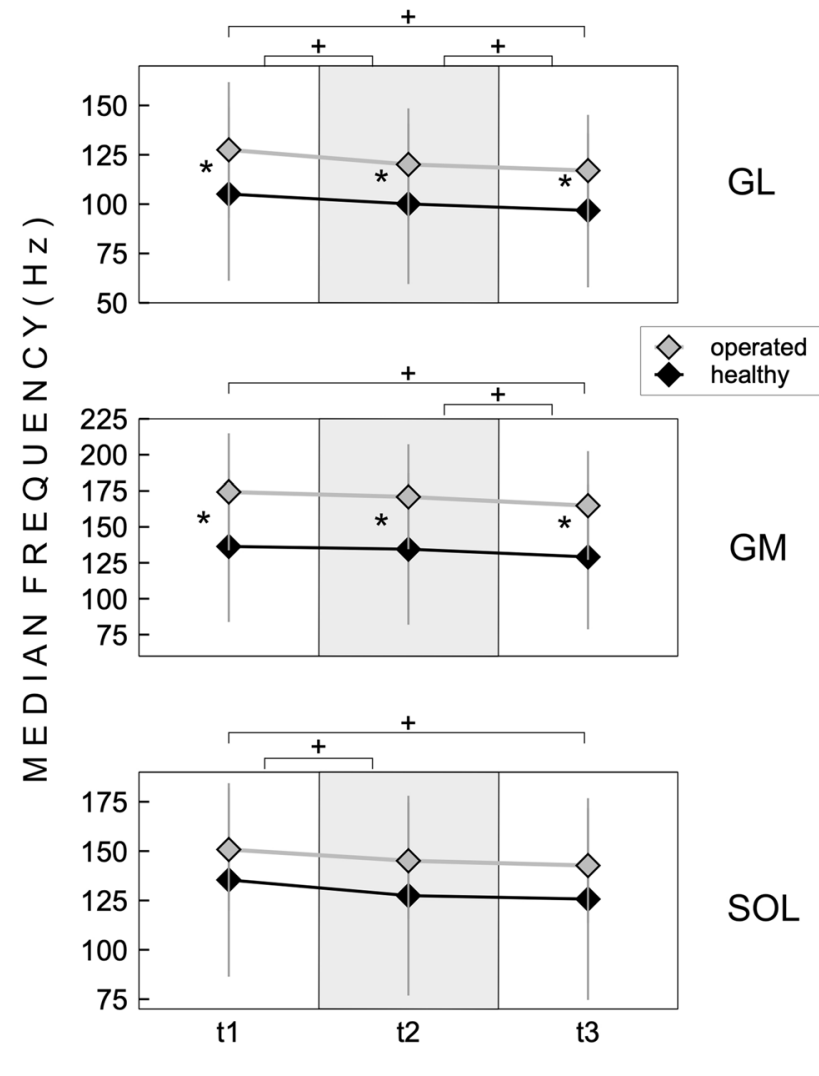

Fig. 3 a Fast Fourier-transformed EMG during static heel-rise showing an overall increased frequency related to an increased recruitment in the activation of type II fibers on the injured side during static conditions. b iEMG during static heel-rise showing an overall increase in

\section{Static heel-rise testing}

As in all testing protocols, we found a higher neuromuscular activation in all time intervals on the affected side, which is in line with preliminary findings in literature [17]. The spectral analysis of EMG activation revealed a significantly higher median firing rate for GM and GL on the injured side, which has been linked to the activation of fast-twitch fiber group $[6,33]$. This observation may either be attributed to a change in structural fiber type from slow-twitch to fasttwitch, which has been described for other injuries [10] or it may result from a different recruiting pattern, where an increased recruiting of type IIa/b fibers is necessary to produce sufficient isometric strength $[3,31]$. The etiology of

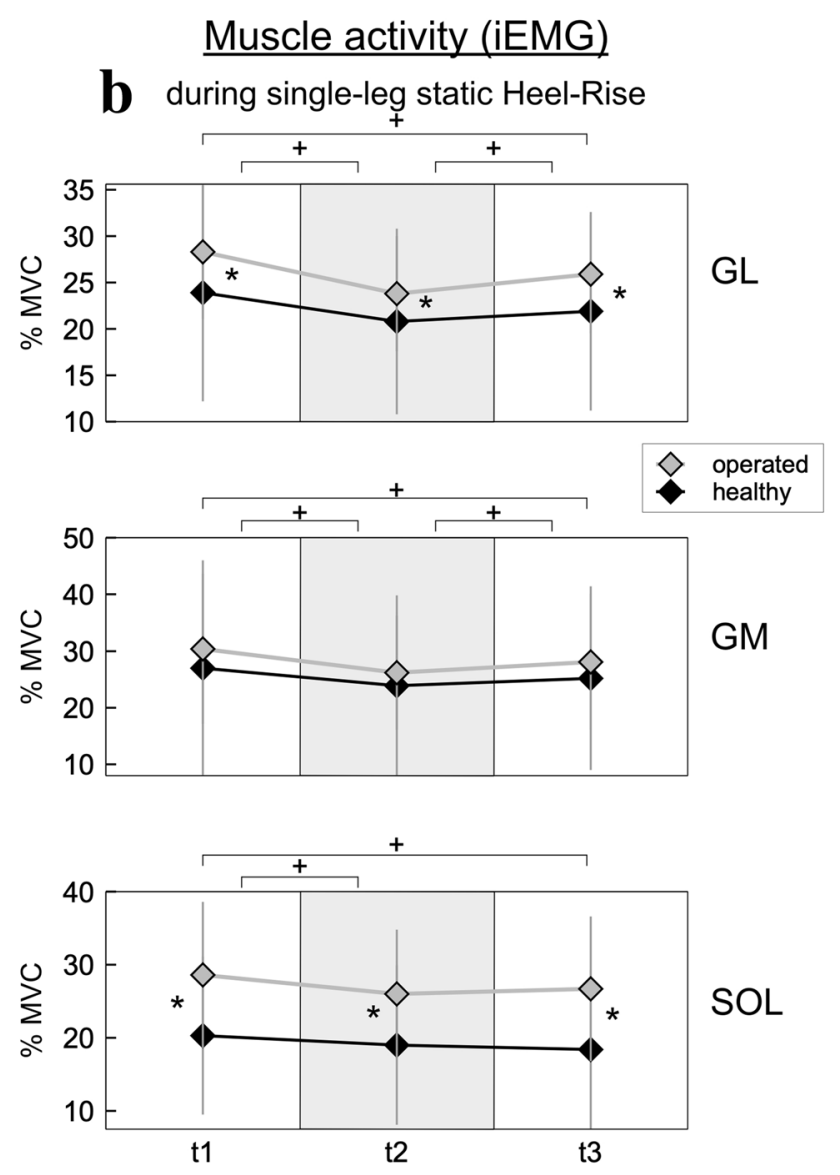

neuromuscular activation integral on the injured side. ${ }^{*} p<0.05$ sideto-side difference, ${ }^{+}$significant differences between time intervals $p<0.05 . t 1=0-2 \mathrm{~s}, t 2=2-8 \mathrm{~s}, t 3=8-10 \mathrm{~s}$

this should be the focus of future research. Regardless of the cause, this may serve as evidence that the contractile potential of the triceps surae muscles is reduced and the muscle could be more prone to fatigue.

\section{Gait analysis}

The finding that the activation level of the plantarflexor muscles during push-off phase is significantly higher may indicate that the energy storage in the injured tendon during the eccentric phase is less effective so that compensatory muscle activation is necessary to produce comparable propulsory force $[1,16,25]$. It can be deducted from the literature that the deficit in tendon recoil work on the injured side must 
Table 3 EMG activation during single-legged static heel-rise testing

\begin{tabular}{lllll}
\hline Muscle & Operated & Non-operated & rm-ANOVA & \\
\cline { 4 - 5 } & & & Factor time & OP vs. NOP \\
\hline iEMG (\% of MVIC) & & & \\
GL 0-2 s & $28.3 \pm 7.2$ & $23.8 \pm 11.7$ & & $p=0.047$ \\
GL 2-8 s & $23.8 \pm 6.2$ & $20.8 \pm 10.0$ & & \\
GL 8-10 s & $25.9 \pm 6.4$ & $21.9 \pm 10.7$ & & n.s. $t 3: p<0.001$ \\
GM 0-2 s & $30.4 \pm 13.2$ & $27.6 \pm 18.9$ & & \\
GM 2-8 s & $26.2 \pm 10.1$ & $23.9 \pm 15.9$ & & \\
GM 8-10 s & $28.0 \pm 12.0$ & $25.2 \pm 16.2$ & & \\
SOL 0-2 sec & $28.6 \pm 10.0$ & $20.2 \pm 10.8$ & $t 1$ vs. $t 2 p<0.001$ & \\
SOL 2-8 s & $26.0 \pm 8.8$ & $19.0 \pm 10.9$ & $t 1$ vs. $t 3 p<0.001$ & \\
SOL 8-10 s & $26.8 \pm 9.9$ & $18.4 \pm 10.9$ & $t 2$ vs. $t 3$ n.s. & \\
Median frequency in Hz (FFT-transformation) & & \\
GL 0-2 s & $127.5 \pm 34.3$ & $105.1 \pm 43.9$ & $t 1$ vs. $t 2$ vs. $t 3: p<0.001$ & \\
GL 2-8 s & $120.1 \pm 28.4$ & $100.1 \pm 40.6$ & & \\
GL 8-10 s & $117.0 \pm 28.2$ & $96.8 \pm 38.9$ & & \\
GM 0-2 s & $174.3 \pm 40.6$ & $136.4 \pm 55.6$ & $t 1$ vs. $t 2$ n.s. & \\
GM 2-8 s & $170.9 \pm 36.4$ & $134.4 \pm 52.6$ & $t 1$ vs. $t 3 p<0.001$ & \\
GM 8-10 s & $164.8 \pm 37.7$ & $129.1 \pm 50.4$ & $t 2$ vs. $t 3 p<0.001$ & \\
SOL 0-2 s & $150.8 \pm 31.4$ & $135.4 \pm 49.0$ & $t 1$ vs. $t 2 p<0.001$ & $t 1$ vs. $t 3 p<0.001$ \\
SOL 2-8 s & $145.1 \pm 29.4$ & $127.4 \pm 50.6$ & $t 2$ vs. $t 3$ n.s. & \\
SOL 8-10 s & $142.7 \pm 28.5$ & $125.7 \pm 51.1$ & & \\
\hline
\end{tabular}

$O P$ operated leg; NOP non-operated leg; $G L$ gastrocnemius lateralis muscle; $G M$ gastrocnemius medialis muscle; $S O L$ soleus muscle; $\% M V C$ in $\%$ of the peak value during maximum voluntary isokinetic contraction; FFT fast Fourier transformation, $2^{n}$ of the median frequency; rm-ANOVA repeated-measures ANOVA, mean \pm standard deviation; $n$.s. non-significant be compensated for by concentric muscular work and, thus, results in a higher activation level compared to the healthy tendon's side $[13,15,19]$.

It is of additional interest that the pre-activation of the tibialis anterior muscle $100 \mathrm{~ms}$ before ground contact is significantly lower on the injured side, while the posterior muscles exhibit the same pre-activation level in both legs. This observation may be attributed to the overall reduced tension of the muscle-tendon unit of the triceps surae muscle and potentially an elongation of the tendon, which consequently requires less activation of the TA to achieve the same joint position at ground contact. However, a recent review found that tendon elongation correlates with biomechanical parameters, but not patient-reported outcome, yielding that this requires further and detailed analysis [8].

Limitations of the study include the retrospective recruitment of patients, which always poses a risk of a recruiting bias. However, we have analyzed the nonrecruited patients according to their biometrical data and we have not found any indication for a recruiting bias.
Further limitations include the novel testing setup and the lack of causal investigation regarding tendon lengthening and stiffness. Thus, we are unable to report the tendon length and stiffness; however, earlier studies have not been able to underscore a correlation between tendon length and neuromuscular activation [25].

\section{Conclusions}

The results of this study provide evidence that there are persisting neuromuscular adaptations 3.5 years after open Achilles tendon repair. These neuromuscular changes are manifested consistently for simple monoarticular up to complex whole-body multiarticular movements. The increased neuromuscular activity, thereby, precludes a primary phenomenon of neural inhibition as part of the performance deficit. The slightly increased muscle co-contraction indicates a neuromuscular stiffing of the joint to reduce the degree of freedom in favor of safety. 
Fig. 4 EMG activation pattern during gait analysis across the three phases. $* p<0.05$

\section{Muscle activation according to phase during gait cycle}
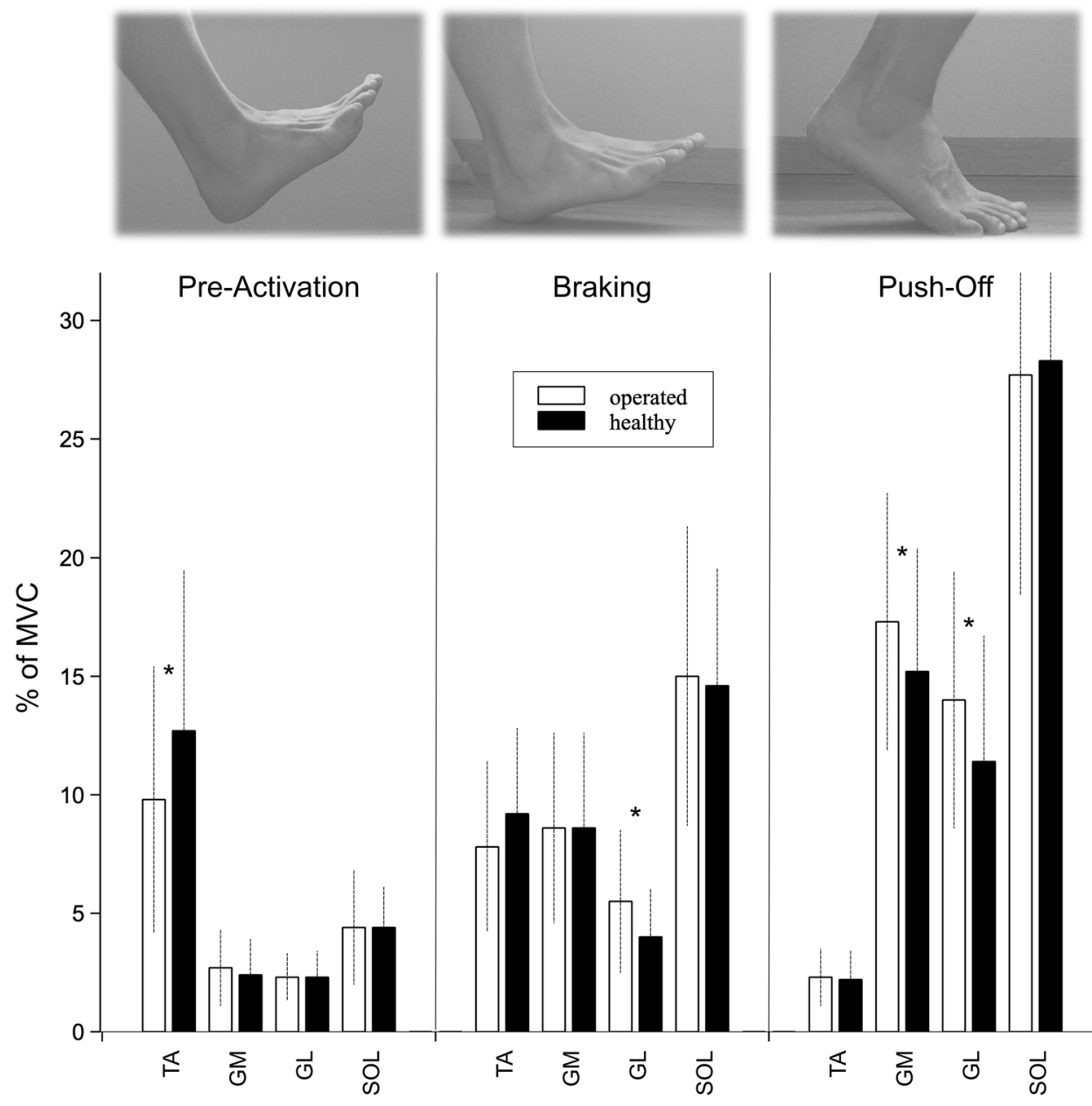

ơ
ANOVA

Operated (\%MVC) Non-

operated

(\%MVC)

\begin{tabular}{llrrl}
\hline TA & Pre-activation & $\mathbf{9 . 8} \pm \mathbf{5 . 6}$ & $\mathbf{1 2 . 7} \pm \mathbf{6 . 8}$ & $\boldsymbol{p}=\mathbf{0 . 0 2 6}$ \\
& Braking & $7.8 \pm 3.6$ & $9.2 \pm 3.6$ & n.s. \\
& Push-off & $2.3 \pm 1.2$ & $2.2 \pm 1.2$ & n.s. \\
GM & Pre-activation & $2.7 \pm 1.6$ & $2.4 \pm 1.5$ & n.s. \\
& Braking & $9.8 \pm 4.0$ & $9.2 \pm 4.0$ & n.s. \\
& Push-off & $\mathbf{1 7 . 3} \pm \mathbf{5 . 4}$ & $\mathbf{1 5 . 2} \pm \mathbf{5 . 2}$ & $\boldsymbol{p}=\mathbf{0 . 0 4 7}$ \\
GL & Pre-activation & $2.3 \pm 1.0$ & $2.3 \pm 1.1$ & n.s. \\
& Braking & $\mathbf{5 . 5} \pm \mathbf{3 . 0}$ & $\mathbf{4 . 0} \pm \mathbf{2 . 0}$ & $\boldsymbol{p}=\mathbf{0 . 0 0 5}$ \\
& Push-off & $\mathbf{1 4 . 0} \pm \mathbf{5 . 4}$ & $\mathbf{1 1 . 4} \pm \mathbf{5 . 4}$ & $\boldsymbol{p}=\mathbf{0 . 0 1 5}$ \\
SOL & Pre-activation & $4.4 \pm 2.4$ & $4.4 \pm 1.7$ & n.s. \\
& Braking & $15.0 \pm 6.3$ & $14.6 \pm 5.5$ & n.s. \\
& Push-off & $27.7 \pm 9.3$ & $29.3 \pm 9.7$ & n.s. \\
\hline
\end{tabular}

Bold values represent significant difference

$T A$ tibialis anterior muscle; $G L$ gastrocnemius lateralis muscle; $G M$ M. gastrocnemius medialis muscle; SOL soleus muscle; $\% M V C$ in $\%$ of the peak value during maximum voluntary isokinetic contraction; ANOVA single-factor ANOVA, factor: side, mean \pm standard deviation
Acknowledgements The first author was supported as a fellow of the Berta-Ottenstein program for Clinician Scientists of the Medical Faculty of the University of Freiburg, Germany. The authors would like to thank Prof. Dr. W. Schlickewei and PD Dr. D. Gehring for their support of the study.

Author contributions All authors have contributed substantially to this study and its submission, and have agreed to the final version of this manuscript.

Funding Open Access funding enabled and organized by Projekt DEAL.

\section{Declarations}

Conflict of interest The authors declare that they have no competing interest.

Funding This study was carried out at and funded by Praxisklinik Rennbahn AG.

Ethical approval This study was approved by the local ethics committee and performed in accordance with the Declaration of Helsinki. All participants declared informed consent prior to inclusion. 
Open Access This article is licensed under a Creative Commons Attribution 4.0 International License, which permits use, sharing, adaptation, distribution and reproduction in any medium or format, as long as you give appropriate credit to the original author(s) and the source, provide a link to the Creative Commons licence, and indicate if changes were made. The images or other third party material in this article are included in the article's Creative Commons licence, unless indicated otherwise in a credit line to the material. If material is not included in the article's Creative Commons licence and your intended use is not permitted by statutory regulation or exceeds the permitted use, you will need to obtain permission directly from the copyright holder. To view a copy of this licence, visit http://creativecommons.org/licenses/by/4.0/.

\section{References}

1. Agres AN, Duda GN, Gehlen TJ, Arampatzis A, Taylor WR, Manegold S (2015) Increased unilateral tendon stiffness and its effect on gait 2-6 years after Achilles tendon rupture. Scand J Med Sci Sports 25:860-867

2. Arslan A, Çepni SK, Sahinkaya T, May C, Mutlu H, Parmaksızoğlu AS (2014) Functional outcomes of repair of Achilles tendon using a biological open surgical method. ActaOrthopTraumatolTurc 48:563-569

3. Bilodeau M, Schindler-Ivens S, Williams DM, Chandran R, Sharma SS (2003) EMG frequency content changes with increasing force and during fatigue in the quadriceps femoris muscle of men and women. J ElectromyogrKinesiol 13:83-92

4. Bohm S, Mersmann F, Santuz A, Arampatzis A (2019) The forcelength-velocity potential of the human soleus muscle is related to the energetic cost of running. Proc R Soc B BiolSci 286:20192560

5. Burden A (2010) How should we normalize electromyograms obtained from healthy participants? What we have learned from over 25 years of research. J ElectromyogrKinesiol 20:1023-1035

6. Chowdhury SK, Nimbarte AD (2015) Comparison of Fourier and wavelet analysis for fatigue assessment during repetitive dynamic exertion. J ElectromyogrKinesiol 25:205-213

7. De la Fuente C, Henriquez H, Carmont MR, Huincahue J, Paredes T, Tapia M, Araya JP, Díaz N, Carpes FP (2021) Do the heelrise test and isometric strength improve after Achilles tendon repair using Dresden technique? Foot Ankle Surg. https://doi. org/10.1016/j.fas.2021.01.007

8. Diniz P, Pacheco J, Guerra-Pinto F, Pereira H, Ferreira FC, Kerkhoffs G (2020) Achilles tendon elongation after acute rupture: is it a problem? A systematic review. Knee Surg Sports TraumatolArthrosc 28:4011-4030

9. Don R, Ranavolo A, Cacchio A, Serrao M, Costabile F, Iachelli M, Camerota F, Frascarelli M, Santilli V (2007) Relationship between recovery of calf-muscle biomechanical properties and gait pattern following surgery for Achilles tendon rupture. ClinBiomech 22:211-220

10. Drechsler WI, Cramp MC, Scott OM (2006) Changes in muscle strength and EMG median frequency after anterior cruciate ligament reconstruction. Eur J ApplPhysiol 98:613-623

11. Henriksen M, Aaboe J, Bliddal H, Langberg H (2009) Biomechanical characteristics of the eccentric Achilles tendon exercise. J Biomech 42:2702-2707

12. Hermens HJ, Freriks B, Disselhorst-Klug C, Rau G (2000) Development of recommendations for SEMG sensors and sensor placement procedures. J ElectromyogrKinesiol 10:361-374

13. Ishikawa M, Komi PV, Grey MJ, Lepola V, Bruggemann G-P (2005) Muscle-tendon interaction and elastic energy usage in human walking. J ApplPhysiol 99:603-608

14. Kastoft R, Bencke J, Speedtsberg MB, Penny JØ, Barfod K (2019) Early weight-bearing in nonoperative treatment of acute Achilles tendon rupture did not influence mid-term outcome: a blinded, randomised controlled trial. Knee Surg Sports TraumatolArthrosc 27:2781-2788

15. Komi PV, IOC Medical Commission, International Federation of Sports Medicine (eds) (2003) Strength and power in sport/edited by Paavo V. Komi. Blackwell Science, Osney Mead, Oxford, ISBN 978-0-632-05911-9

16. Laurent D, Walsh L, Muaremi A, Beckmann N, Weber E, Chaperon F, Haber H, Goldhahn J, Klauser AS, Blauth M, Schieker M (2020) Relationship between tendon structure, stiffness, gait patterns and patient reported outcomes during the early stages of recovery after an Achilles tendon rupture. Sci Rep 10:20757

17. McHugh MP, Orishimo KF, Kremenic IJ, Adelman J, Nicholas SJ (2019) Electromyographic evidence of excessive Achilles tendon elongation during isometric contractions after Achilles tendon repair. Orthop J Sports Med. https://doi.org/10.1177/2325967119 883357

18. Nilsson-Helander K, GrävareSilbernagel K, Thomeé R, Faxén E, Olsson N, Eriksson BI, Karlsson J (2010) Acute Achilles tendon rupture: a randomized, controlled study comparing surgical and nonsurgical treatments using validated outcome measures. Am J Sports Med 38:2186-2193

19. Novacheck T (1998) The biomechanics of running. Gait Posture 7:77-95

20. Oda H, Sano K, Kunimasa Y, Komi PV, Ishikawa M (2017) Neuromechanical modulation of the Achilles tendon during bilateral hopping in patients with unilateral Achilles tendon rupture, over 1 year after surgical repair. Sports Med 47:1221-1230

21. Okoroha KR, Ussef N, Jildeh TR, Khalil LS, Hasan L, Bench C, Zeni F, Eller E, Moutzouros V (2020) Comparison of tendon lengthening with traditional versus accelerated rehabilitation after Achilles tendon repair: a prospective randomized controlled trial. Am J Sports Med. https://doi.org/10.1177/0363546520909389

22. Sharma P, Maffulli N (2005) Tendon injury and tendinopathy: healing and repair. J Bone JtSurg 87:187-202

23. Silbernagel KG, Steele R, Manal K (2012) Deficits in heel-rise height and Achilles tendon elongation occur in patients recovering from an Achilles tendon rupture. Am J Sports Med 40:1564-1571

24. Souissi H, Zory R, Bredin J, Gerus P (2017) Comparison of methodologies to assess muscle co-contraction during gait. J Biomech 57:141-145

25. Speedtsberg MB, Kastoft R, Barfod KW, Penny JØ, Bencke J (2019) Gait function and postural control 4.5 years after nonoperative dynamic treatment of acute Achilles tendon ruptures. Orthop J Sports Med. https://doi.org/10.1177/2325967119854324

26. Suydam SM, Buchanan TS, Manal K, Silbernagel KG (2015) Compensatory muscle activation caused by tendon lengthening post-Achilles tendon rupture. Knee Surg Sports TraumatolArthrosc 23:868-874

27. Tengman T, Riad J (2013) Three-dimensional gait analysis following Achilles tendon rupture with nonsurgical treatment reveals long-term deficiencies in muscle strength and function. Orthop J Sports Med 1:232596711350473

28. Trofa DP, Miller JC, Jang ES, Woode DR, Greisberg JK, Vosseller JT (2017) Professional athletes' return to play and performance after operative repair of an Achilles tendon rupture. Am J Sports Med 45:2864-2871

29. Trofa DP, Noback PC, Caldwell J-ME, Miller JC, Greisberg JK, Ahmad CS, Vosseller JT (2018) Professional soccer players' return to play and performance after operative repair of Achilles tendon rupture. Orthop J Sports Med 6:232596711881077

30. Von Tscharner V, Goepfert B (2006) Estimation of the interplay between groups of fast and slow muscle fibers of the tibialis anterior and gastrocnemius muscle while running. J ElectromyogrKinesiol 16:188-197 
31. Warren GL, Hermann KM, Ingalls CP, Masselli MR, Armstrong RB (2000) Decreased EMG median frequency during a second bout of eccentric contractions. Med Sci Sports Exerc 32:820-829

32. Wenning M, Mauch M, Heitner A, Streicher P, Ritzmann R, Paul J (2021) Midterm functional performance following open surgical repair of acute Achilles tendon rupture. Arch Orthop Trauma Surg. https://doi.org/10.1007/s00402-020-03746-3

33. Winter DA (2009) Biomechanics and motor control of human movement. Wiley, Hoboken, pp 1-370
34. Zellers JA, Marmon AR, Ebrahimi A, GrävareSilbernagel K (2019) Lower extremity work along with triceps surae structure and activation is altered with jumping after Achilles tendon repair. J Orthop Res 37:933-941

Publisher's Note Springer Nature remains neutral with regard to jurisdictional claims in published maps and institutional affiliations. 